\title{
Rigid-Plastic Analysis of Seismic Resistant T-Frame considering Moment-Shear Interaction
}

\author{
Ghader Bagheri $\mathbb{D}^{1},{ }^{1}$ Payam Ashtari $\mathbb{D}{ }^{2}$ and Farhad Behnamfar $\mathbb{D}^{3}$ \\ ${ }^{1}$ Department of Civil Engineering, Isfahan (Khorasgan) Branch, Islamic Azad University, Isfahan, Iran \\ ${ }^{2}$ Department of Civil Engineering, University of Zanjan, Zanjan, Iran \\ ${ }^{3}$ Department of Civil Engineering, Isfahan University of Technology, Isfahan, Iran
}

Correspondence should be addressed to Ghader Bagheri; gh.bagheri@iauz.ac.ir

Received 17 September 2020; Revised 6 June 2021; Accepted 20 June 2021; Published 6 July 2021

Academic Editor: Paola Forte

Copyright (C) 2021 Ghader Bagheri et al. This is an open access article distributed under the Creative Commons Attribution License, which permits unrestricted use, distribution, and reproduction in any medium, provided the original work is properly cited.

\begin{abstract}
To select a seismic resistant system, in addition to strength and stiffness, ductility and energy dissipation are important to be considered. Structures have nonlinear behavior under the influence of moderate and strong earthquakes. One of the primary aims in designing seismic resistant structures is to prevent the formation of undesirable collapse mechanisms such as the collapse in only a few storeys of the structure that leads to low energy dissipation. In order to achieve a global collapse mechanism, modern seismic codes provide simple rules for design, which is called the hierarchy criteria. Although these simple criteria could prevent the formation of a soft storey mechanism, they could not lead to an optimal global collapse mechanism. In these mechanisms, the energy dissipation zones include all the yielding zones such as beams, while all other parts of the structure have remained in the elastic range. TRF (T-resisting frame) is an innovative lateral resistant system introduced for architectural reasons and to provide more energy dissipating capability. This system has several collapse mechanisms due to the moment, shear, or moment-shear behavior of its members. In this paper, within the framework of the theory of plastic mechanism control, the rigid-plastic analysis of the TRF system to achieve the desired collapse mechanism is used by considering the moment-shear interaction. According to these analyses, which are performed on a single storey frame, simple hierarchy criteria are developed to create the desired collapse mechanism. Also, these criteria prevent undesired collapse mechanisms in order to have more energy dissipation and more ductility. Finally, the validity of the proposed criteria has been verified by the pushover analysis.
\end{abstract}

\section{Introduction}

T-resisting frame is a new lateral force resisting system, which consists of two structural parts, T-part as the main component and the second part of side columns. In steel structures, T-part is composed of a vertical plate girder (VPG) and a pair of horizontal plate girders (HPGs) to provide significant lateral stiffness and high energy dissipation capability under severe earthquakes. TRF dissipates energy predominantly through stable shear yielding of the web shear panels of HPGs. Also, side columns in TRF, such as columns in conventional braced frames, withstand compression or tension to resist the overturning moments (Figure 1) [1].

Ashtari proposed the configuration of TRF in 2008 and Bandehzadeh in the M.Sc thesis demonstrated the seismic performance advantages of the optimized single-T TRFs [1].
Ashtari (2008) to provide and improve building's behavior and performance presented the initial idea of this lateral seismic system. Initial numerical and experimental studies have been conducted on the behavior and performance of the TRF system by Ashtari et al. [1,2-5]. The results of nonlinear finite element analyses in this system demonstrate that TRF has high initial stiffness, high ductility, excellent energy dissipation capacity, and stable hysteretic behavior at large inelastic deformations under numerous cycles of loading. According to the results from a series of nonlinear analyses, a properly designed TRF exhibited a great efficiency as the lateral resistant system of a building in a high seismic zone due to its potential for extremely high ductility and energy dissipating capability. In this building, the observed drifts and rotation demands were significantly lower than those commonly associated with the buildings using common moment-resisting frames [1]. Various 


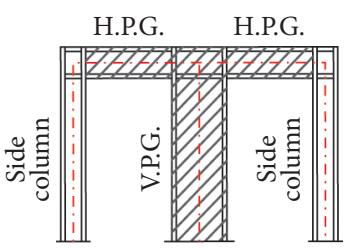

(a)

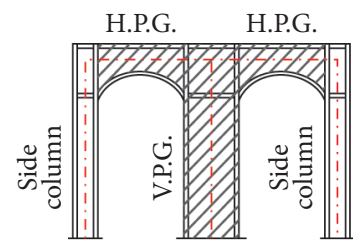

(d)

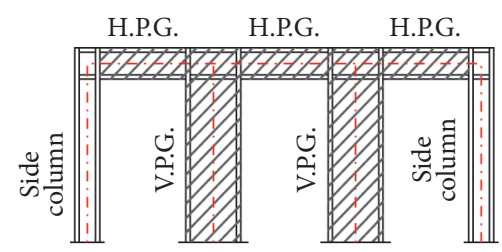

(b)

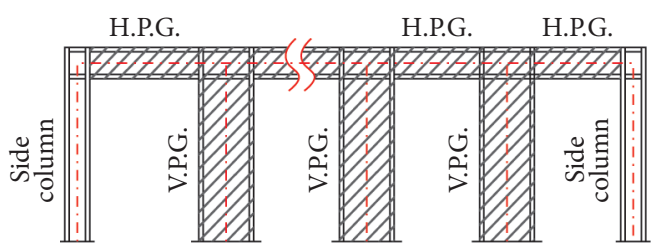

(c)

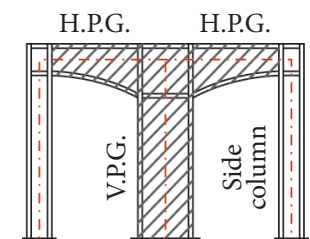

(e)

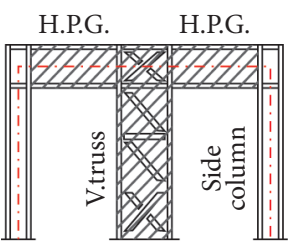

(f)

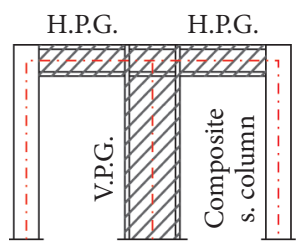

(g)

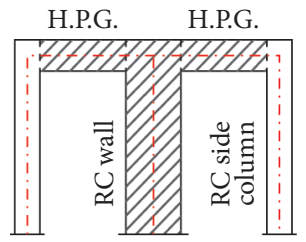

(h)

Figure 1: Proposed basic structural configurations of TRF and alternatives [1]. (a) Single-T configuration of TRF. (b) Double-T configuration of TRF. (c) Multi-T configuration of TRF. (d) Stylish single-T configuration of TRF. (e) Stylish single-T configuration of TRF. (f) Single-T configuration of TRF using vertical truss. (g) Single-T configuration of TRF using composite side columns. (h) Single-T configuration of reinforced concrete TRF.

numerical studies and laboratory tests have been performed on different configurations of this system. The results show that this system has good seismic behavior. The results show that this system has high initial stiffness, high ductility, excellent energy dissipation capacity, and stable hysteretic behavior at large inelastic deformations under numerous cycles of loading $[4,5]$.

In the capacity design, the energy dissipation zones should be designed according to the internal forces resulting from the load combinations provided by the seismic codes. The other parts of the structure have to be designed proportionally to the force of the energy dissipation zones. It means that these parts do not yield under the maximum internal reactions transmitted by the energy dissipation zones in the fully yielded state $[6,7]$.

In the eccentrically braced frames, dissipative zones are constituted by the members called link elements [8-10]. The use of members as energy dissipation zones, which they are yielding in shear, was originally proposed for eccentrically braced frames. This issue has been the starting point for the formation of new structural types such as knee braces [11], aluminum shear links [12], and shear links made of low-flow point steel [13]. Shear yielding is separated from the energy dissipation due to moment yielding, so the moment-shear interaction could not be neglected.

In this paper, the TRF-H system has been studied, in which the task of energy dissipation is assigned to the T-part members as link elements, which are affected by moment, shear, or moment-shear interaction. The problem of moment-shear interaction is considered within the framework of rigid-plastic analysis and the solution for the TRF-H system is presented. Solving the interaction problem is affected by compatibility conditions, which depend on the configuration of the structure. Such issue is currently being studied for eccentrically braced frames with D-scheme, $\mathrm{K}$-scheme, V-scheme, inverted V-scheme [14], and Y EB-
Frames [15], and here for the T-resisting frame system. The purpose of developing the hierarchy criteria is to control the yielding pattern. Investigations are focused on the onestorey TRF-H system to determine which members and how they yield. This theory is based on the rigid-plastic analysis with the purpose of achieving the global collapse mechanism, in which all the dissipation zones are yielding, while the other members stay in the elastic range. This theory has been presented for frames with horizontal link beam [16], knee braces (KB-frame) [17], dual MRF-CBF systems [18], and truss moment frame (DTMFs) [19]. Also, the solution of the theory of plastic mechanism control (TPMC) currently being studied in the case of moment-resisting frames: eccentrically braced frames (MRF-EBF) dual systems [20] and eccentrically braced frames with inverted Y-scheme [21]. These studies provide a complete and exhaustive design procedure for MRF-EBF dual systems and EB-frames with inverted $\mathrm{Y}$-scheme, considering all the brace configurations commonly adopted and with the goal of assuring the development of a collapse mechanism of global type [20,21].

In Section 2, the study of moment-shear interaction in the TRF-H system and the development of the hierarchy criteria at the storey level have been studied. To assess the accuracy of the proposed hierarchy criteria for the TRF-H system has been examined by means of static pushover analyses with the SAP2000 computer program. In addition, the theory of controlling plastic mechanisms for the multistorey TRF system and fixed multistorey TRF system would be provided in subsequent papers.

\section{The Plastic Mechanisms in the TRF-H System}

Independent from the configuration of the structure, the main feature of the links is due to the interaction between the bending moment and the shear force, which could not be ignored in predicting the ultimate behavior of the structural 
system. Generally, the classification of the link beams depends on the length of the member. In the following, general classification is proposed [7-10]:

(i) Short link for $e \leq 1.6 M_{P} / V_{P}$

(ii) Intermediate link for $1.6 M_{P} / V_{P} \leq e \leq 3 M_{P} / V_{P}$

(iii) Long links for $e \geq 3 M_{P} / V_{P}$

$V_{p}$ and $M_{p}$, respectively, are plastic shear strength and bending strength of the cross section of the link beam. The long link beam is considered to be in frequent revisions as $2.6 M_{P} / V_{P}$ [22-24]. Moment-shear interaction diagram and the short, medium, and long links' range are shown in Figure 2.

The plastic behavior of the link beam, for a bending moment less than $M_{f}$, could be considered as a pure shear, where $M_{f}$ is the flange contribution of the plastic moment of the link beam; thus, for moments greater than $M_{f}$, the shear strength of the element is reduced due to the interaction between moment and shear. According to Neal research, the plastic zone is calculated using the following relationships $[14,25]$ :

$$
\begin{aligned}
\left(\frac{|M|-M_{f}}{M_{p}-M_{f}}\right)^{2}+\left(\frac{V}{V_{p}}\right)^{2} & =1, \quad \text { for } M_{f} \leq|M| \leq M_{P}, \\
V & =V_{p}, \quad \text { for }|M| \leq M_{f} .
\end{aligned}
$$

In capacity design, an important issue that has been reported in previous experiments by Hjelmstad and Popov [26], and Malley and Popov [27] is the overstrength developed by the link beam. Overstrength of the link beam is the ratio between the maximum shear forces developed by the link beam to its plastic shear strength. The evaluation of the results of experiments is usually based on the inclusion of the plastic shear strength as $V_{P}=0.6 f_{y}\left(d-t_{f}\right) t_{w}$, where $f_{y}$ is the yield stress, $d$ is the depth of the cross section of the beam, and $t_{f}$ and $t_{w}$ are the thickness of the flange and the web, respectively. However, the link overstrength occurs due to the strain hardening and the contribution of link flanges to the shear strength. Therefore, according to European codes [28], plastic shear strength is calculated using the web surface shear strength with the contribution of the flange.

According to the capacity design, the link overstrength should be calculated in the assessment of the maximum internal forces transferred to the nonplastic elements. The link overstrength factor is generally recommended as 1.50 , based on previous experimental studies [29]. In the 2005 AISC seismic provisions [23], the link overstrength factor is proposed equal to 1.25 for designing the diagonal braces and equal to 1.10 for designing columns and the beam outside the link. A similar approach has been adopted in the eurocode [6]. Recent experimental results and investigations are indicated that overstrength factors can be exceeding the code suggested values. For very short links, with compact cross-sections and perfect axial restraints, values of shear overstrength up to 2 have been obtained. For built-up links with a very compact shape and short

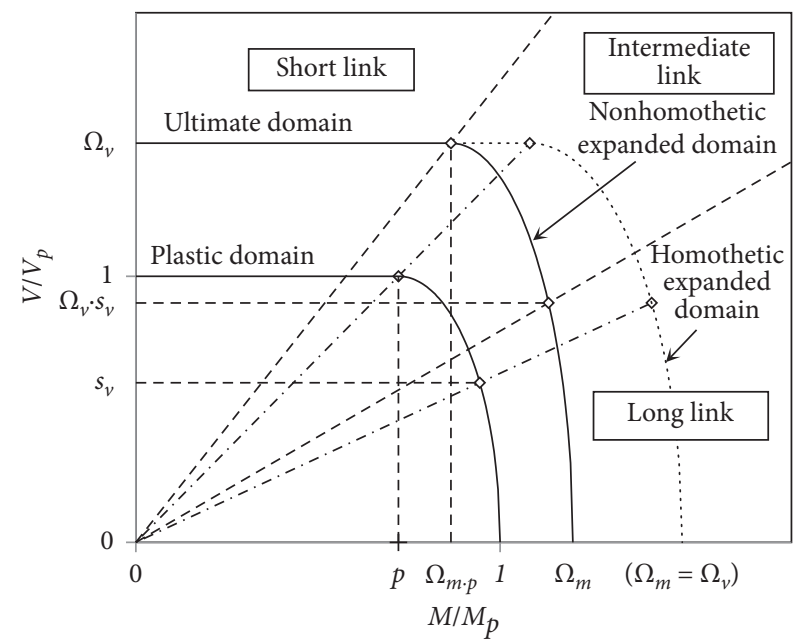

Figure 2: Moment-shear interaction diagram $\left(p=M_{f} / M_{p}\right)$.

length, even larger values could be obtained, especially if larger rotation capacity is achievable [30]. Barecchia et al., by means of finite element analyses on standard European rolled profiles, have found overstrength factors between 2.00 and 2.50 for a link shear deformation equal to $0.10 \mathrm{rad}$ [31]. Nevertheless, in using the above results, some caution should be adopted because they come from finite element analyses where the stiffeners' influence cannot be calculated accurately.

Recently, Okazaki et al., based on several experimental tests on shear links with properly detailed web stiffener, have obtained the overstrength factor between 1.34 and 1.48 for short links and in the range of 1.12-1.28 for longer links [24]. These experimental results of the overstrength factor have been computed considering the measured values of the material properties. Therefore, based on these experimental results, it was concluded that the overstrength factor of 1.50, which forms worldwide the basis of capacity design provisions for EB-frames and appears reasonable for links made from rolled profiles. A simple method for calculating the overstrength of the hierarchy criteria obtained by the rigid-plastic analysis has recently been presented [14]. It is based on the use of the interaction domain referring to the ultimate conditions obtained from the homothetic or nonhomothetic expansion of the plastic domain (Figure 2).

The topologies of the plastic collapse mechanisms of the one-storey TRF-H are shown in Figure 3. In the type " $A$ " mechanism, ends of the both horizontal links and the bottom of the central column yield. In mechanism " $B$," two ends of the central column yield. Type of collapse mechanism is determined by the elements and structure specification. The aim of the research presented here is to define the hierarchy criteria, which includes the effect of the momentshear interaction, to ensure that the desired mechanism would be developed.

In fact, in order to calculate the moment and shear interaction in the framework of rigid-plastic analysis, the end-link yield is modeled with a combination of a pair of plastic double pendulum and a plastic hinge that can 


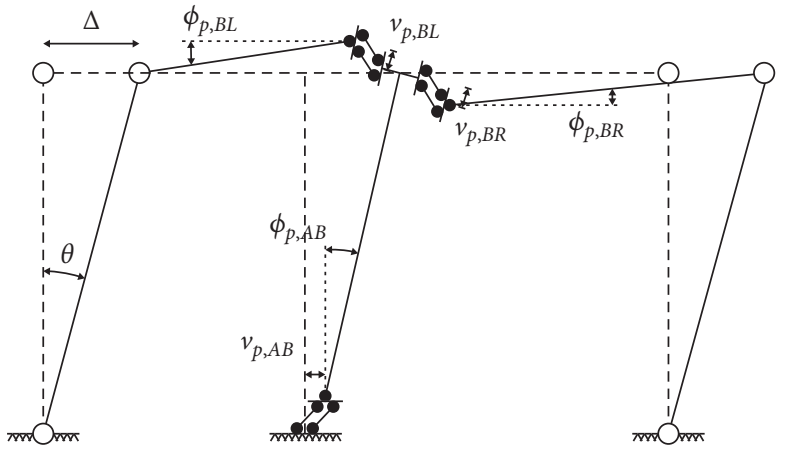

(a)

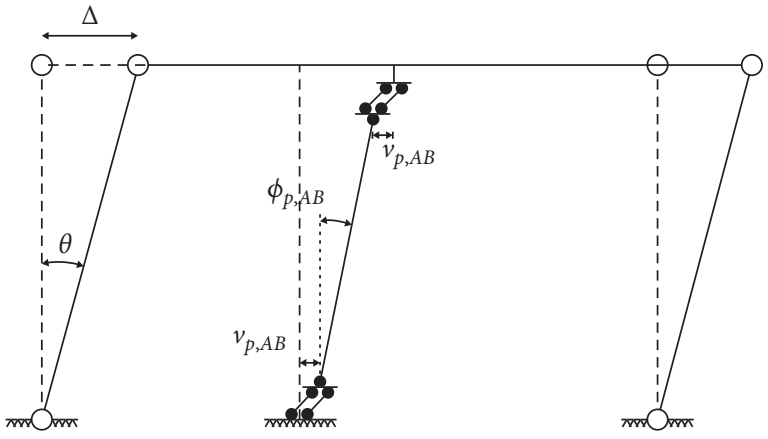

(b)

FIgURe 3: Modeling TRF-H system by considering the moment and shear interaction. (a) Mechanism "A." (b) Mechanism "B."

calculate both types of plastic shear deformation $v_{P}$ and moment plastic rotation $\phi_{p}$, respectively.

Use of the rigid-plastic analysis to calculate the plastic strength of possible mechanisms needs to determine the plastic deformations of the links $\nu_{P}$ and $\phi_{p}$. Also, the values of shear force $V$ and corresponding moment $M$ for a given value of the $\theta$ parameter is needed. Problem solving could be done by minimizing the internal work of the link and applying the yielding conditions and considering of the normal plastic flow rule. Additionally, compatibility conditions should be satisfied.

\section{Moment-Shear Interaction in TRF-H System Elements}

In the equations, the index of parameters is based on the member's names, meaning the $B L$ and $B R$ indexes are related to the left and right horizontal link (beam), respectively, and the $A B$ is related to the vertical member (central column).

3.1. Mechanism Type " $A$ ". Within the framework of rigidplastic analysis, moment-shear interaction occurring in the link of EB-frames is provided in the prior studies [15]. The moment and shear interaction in the elements of the TRF-H frame (Figure 3(a)), within the framework of the rigidplastic analysis, is obtained by the following relationships (equations (3)-(10)):

(1) Minimizing the internal work $W_{i}$ according to the collapse mechanism under the influence of the plastic rotation $\theta$ at the columns, for a hinge state, is

$$
\text { Min. : } W_{i}^{(A)}=M_{B L}^{(A)}\left(\phi_{p, B L}^{(A)}+\phi_{p, A B}^{(A)}\right)+V_{B L}^{(A)} v_{p, B L}^{(A)}+M_{B R}^{(A)}\left(\phi_{p, B R}^{(A)}+\phi_{p, A B}^{(A)}\right)+V_{B R}^{(A)} v_{p, B R}^{(A)}+M_{A B}^{(A)} \phi_{p, A B}^{(A)}+V_{A B}^{(A)} v_{p, A B}^{(A)}
$$

(2) Kinematic compatibility conditions are

$$
\begin{aligned}
& v_{p, B L}^{(A)}+\phi_{p, B L}^{(A)} e_{L}=0, \\
& v_{p, B R}^{(A)}+\phi_{p, B R}^{(A)} e_{R}=0, \\
& v_{p, A B}^{(A)}+\phi_{p, A B}^{(A)} h=\theta h .
\end{aligned}
$$

(3) Elements' yield condition leads to these three functions:

$$
\begin{aligned}
& F\left(M_{B L}^{(A)}, V_{B L}^{(A)}\right)=\left(\frac{\left|M_{B L}^{(A)}\right|-M_{f, b}}{M_{p, b}-M_{f, b}}\right)^{2}+\left(\frac{V_{B L}^{(A)}}{V_{p, b}}\right)^{2}-1, \\
& F\left(M_{B R}^{(A)}, V_{B R}^{(A)}\right)=\left(\frac{\left|M_{B R}^{(A)}\right|-M_{f, b}}{M_{p, b}-M_{f, b}}\right)^{2}+\left(\frac{V_{B R}^{(A)}}{V_{p, b}}\right)^{2}-1,
\end{aligned}
$$

$$
F\left(M_{A B}^{(A)}, V_{A B}^{(A)}\right)=\left(\frac{\left|M_{A B}^{(A)}\right|-M_{f, c}}{M_{p, c}-M_{f, c}}\right)^{2}+\left(\frac{V_{A B}^{(A)}}{V_{p, c}}\right)^{2}-1 .
$$

(4) Normal plastic flow law gives

$$
\begin{aligned}
& v_{p}^{(A)}=\lambda \frac{\partial F}{\partial V^{(A)}}, \\
& \phi_{p}^{(A)}=\lambda \frac{\partial F}{\partial M^{(A)}} .
\end{aligned}
$$

Here, $M^{(A)}$ and $V^{(A)}$ are the moment and the shear formed at the end of TRF-H elements, respectively. $\phi_{P}^{(A)}$ and $v_{P}^{(A)}$ are the plastic rotation and plastic shear deformation occurring in the mechanism " $A$," respectively. $e$ is length of the link, $h$ is height of the storey, and $\theta$ is the plastic rotation at the end of the beam and columns. The " $b$ " and " $c$ " index are for the TRF-H horizontal link beam and central column, respectively. For each 
member of the TRF-H system, the values of the shear and the moment would be determined using the above equations. These calculations are made for the left horizontal link $(B L)$, the right horizontal link $(B R)$, and the central column of the TRF$\mathrm{H}(A B)$ system. For the $B L$ member, by substituting equations (4)-(6) into equation (3), an equation will be obtained that expresses the internal work as a function of the plastic rotation of the T-part's members $\left(\phi_{p}\right)$. So, minimizing the internal work of each member could be expressed as

$$
\begin{aligned}
& \frac{d W_{i}^{(A)}}{d \phi_{p, B L}^{(A)}}=0 \longrightarrow V_{B L}^{(A)}=\frac{M_{B L}^{(A)}}{e_{L}}, \\
& \frac{d W_{i}^{(A)}}{d \phi_{p, B R}^{(A)}}=0 \longrightarrow V_{B R}^{(A)}=\frac{M_{B R}^{(A)}}{e_{R}}, \\
& \frac{\mathrm{d} W_{i}^{(A)}}{\mathrm{d} \phi_{p, A B}^{(A)}}=0 \longrightarrow V_{A B}^{(A)}=\frac{M_{B L}^{(A)}+M_{B R}^{(A)}+M_{A B}^{(A)}}{h} .
\end{aligned}
$$

By defining the plastic moment of the web as $M_{w}$ $\left(M_{w}=M_{p}+M_{f}\right)$ and by combining equations (7)-(9) with equations (11)-(13), for all T-part members, the value of $M^{(A)}$ and $V^{(A)}$ could be determined.
The value of $M^{(A)}$ and $V^{(A)}$ for the left horizontal link $(B L)$ is determined as follows:

$$
\begin{gathered}
M_{B L}^{(A)}=\frac{M_{f, b}+M_{w, b} \sqrt{1-\left(\left(M_{f, b}^{2}-M_{w, b}^{2}\right) / V_{p, b}^{2} e_{L}^{2}\right)}}{1+\left(M_{w, b}^{2} / V_{p, b}^{2} e_{L}^{2}\right)}, \\
V_{B L}^{(A)}=\frac{M_{f, b}+M_{w, b} \sqrt{1-\left(\left(M_{f, b}^{2}-M_{w, b}^{2}\right) / V_{p, b}^{2} e_{L}^{2}\right)}}{e_{L}\left(1+\left(M_{w, b}^{2} / V_{p, b}^{2} e_{L}^{2}\right)\right)} .
\end{gathered}
$$

By defining the parameter $p_{b}=M_{f, b} / M_{p, b}$, the moments can be rewritten based on the beam's plastic moment. Accordingly, by substituting the values of in $M_{f, b}=p_{b} M_{p, b}$ and $M_{w, b}=\left(1-p_{b}\right) M_{p, b}$ and $V_{p, b}=\left(4\left(1-p_{b}\right) / \sqrt{3} h_{w, b}\right)$ $M_{p, b}$ into equation (14), the $B L$ moment is defined as the beam plastic moment coefficient as follows:

$$
\begin{gathered}
M_{B L}^{(A)}=x_{B L}^{(A)} \cdot M_{p, b}, \\
V_{B L}^{(A)}=\frac{x_{B L}^{(A)}}{e_{L}} \cdot M_{p, b} .
\end{gathered}
$$

In these equations, the multiplier $x_{B L}^{(A)}$ is equal to

$$
x_{B L}^{(A)}=\frac{p_{b}+\left(1-p_{b}\right) \sqrt{1-(3 / 16)\left(h_{w, b} / e_{L}\right)^{2}\left(\left(2 p_{b}-1\right) /\left(1-p_{b}\right)^{2}\right)}}{1+(3 / 16)\left(h_{w, b} / e_{L}\right)^{2}}, \quad p_{b} \leq x_{B L}^{(A)} \leq 1 .
$$

Using the above equations, with the definition of the shear length in the form of $e_{v}=M_{f, b} / V_{p, b}$, for different shear values $V_{B L}^{(A)}=s_{v, b} \cdot V_{p, b}$ (Figure 1), link length and moment factor $\left(x_{B L}^{(A)}\right)$ can be obtained as follows:

$$
\begin{aligned}
e_{L} & =\left(\frac{p_{b}+\left(1-p_{b}\right) \sqrt{1-s_{b}^{2}}}{p_{b} s_{b}}\right) e_{v}, \\
x_{B L}^{(A)} & =p_{b}+\left(1-p_{b}\right) \sqrt{1-s_{b}^{2}} .
\end{aligned}
$$

According to the above equations, for $s_{v, b}=1$, equations (19) and (20) conclude that $e_{L}=e_{v}$ and $x_{B L}^{(A)}=p_{b}$, respectively. Consequently, using equation (16), we have $M_{B L}^{(A)}=M_{f, b}$, which indicates a short (shear) link. In addition, where
$e_{L} \longrightarrow \infty$, equation (18) concludes that $x_{B L}^{(A)}=1$. So, according to equation (16), we would have $M_{B L}^{(A)}=M_{p, b}$ and equation (17) gives $V_{B L}^{(A)}=M_{p, b} / e_{L}$, which this state indicates the long (moment) link. Also, the values of $M_{B L}^{(A)}$ and $V_{B L}^{(A)}$ could be determined simply by combining the equilibrium conditions in the link with the yielding condition. In other words, the solution obtained is also reliable in a static state so that it provides the actual internal reactions under the yielding conditions in moment-shear interactions.

In addition, using these equations and choosing the value of $s_{v, b}$, the length of the long link could also be determined. According to AISC99 and AASHTO, if this value is considered 0.6 , then we have $[32,33]$

$$
\left\{\begin{array}{l}
e_{m}=\left(\frac{p_{b}+4}{3 p_{b}}\right) e_{v}, \\
x_{B L}^{(A)}=0.2 p_{b}+0.8, \\
M_{B L}^{(A)}=\left(0.2 p_{b}+0.8\right) M_{p, b} \stackrel{0<p_{b}<1}{\longrightarrow} M_{B L}^{(A)}=(0.8 \sim 1.0) M_{p, b} \text { (long link). }
\end{array}\right.
$$


In this relation, $e_{m}$ is defined as the length of moment (long) link.
Also, for the right beam $(B R)$, it can be determined as follows:

$$
\begin{aligned}
& M_{B R}^{(A)}=\frac{M_{f, b}+M_{w, b} \sqrt{1-\left(\left(M_{f, b}^{2}-M_{w, b}^{2}\right) / V_{p, b}^{2} e_{R}^{2}\right)}}{1+\left(M_{w, b}^{2} / V_{p, b}^{2} e_{R}^{2}\right)}, \\
& V_{B R}^{(A)}=\frac{M_{f, b}+M_{w, b} \sqrt{1-\left(\left(M_{f, b}^{2}-M_{w, b}^{2}\right) / V_{p, b}^{2} e_{R}^{2}\right)}}{e_{R}\left(1+\left(M_{w, b}^{2} / V_{p, b}^{2} e_{R}^{2}\right)\right)}, \\
& x_{B R}^{(A)}=\frac{p_{b}+\left(1-p_{b}\right) \sqrt{1-(3 / 16)\left(h_{w, b} / e_{R}\right)^{2}\left(\left(2 p_{b}-1\right) /\left(1-p_{b}\right)^{2}\right)}}{1+(3 / 16)\left(h_{w, b} / e_{R}\right)^{2}}, \\
& M_{B R}^{(A)}=x_{B R}^{(A)} \cdot M_{p, b}, \\
& V_{B R}^{(A)}=\frac{x_{B R}^{(A)}}{e_{R}} \cdot M_{p, b} .
\end{aligned}
$$

In this case, for a link with long length $\left(e_{R} \longrightarrow \infty\right)$, according to equation (24) $x_{B R}^{(A)}=1$. Consequently, by using equations (25) and (26), we have $M_{B R}^{(A)}=M_{p, b}$ and $V_{B R}^{(A)}=M_{p, b} / e_{R}$, respectively, which indicates the long link. All the relations and conditions examined for the left link $(B L)$ are confirmed for the right link $(B R)$.
Finally, for the central column $(A B)$ by defining the parameter $p_{c}=M_{f, c} / M_{p, c}$ and by substituting the values $M_{p, c}=z \cdot M_{p, b}, \quad M_{f, c}=p_{c} M_{p, c}, \quad M_{w, c}=\left(1-p_{c}\right) M_{p, c}$, and $V_{p, c}=\left(\left(4\left(1-p_{c}\right)\right) / \sqrt{3} h_{w, c}\right) M_{p, c}$, we have

$$
\begin{aligned}
M_{A B}^{(A)}= & x_{A B}^{(A)} \cdot M_{p, c}, \\
V_{A B}^{(A)}= & \frac{x_{B L}^{(A)}+x_{B R}^{(A)}+z \cdot x_{A B}^{(A)}}{z \cdot h} \cdot M_{p, c}, \\
x_{A B}^{(A)}= & \frac{p_{c}-\left(\left(x_{B L}^{(A)}+x_{B R}^{(A)}\right) / z\right)(3 / 16)\left(h_{w, c} / h\right)^{2}}{\left(1+(3 / 16)\left(h_{w, c} / h\right)^{2}\right)} \\
& +\frac{\left(1-p_{c}\right) \sqrt{1-\left(\left(2 p_{c}+\left(x_{B L}^{(A)}+x_{B R}^{(A)}\right) / z\right)\left(\left(x_{B L}^{(A)}+x_{B R}^{(A)}\right) / z \cdot\left(1-p_{c}\right)^{2}\right)+\left(\left(2 p_{c}-1\right) /\left(1-p_{c}\right)^{2}\right)\right)(3 / 16)\left(h_{w, c} / h\right)^{2}}}{\left(1+(3 / 16)\left(h_{w, c} / h\right)^{2}\right)} .
\end{aligned}
$$

According to equation (27), moment of central column can be defined as a coefficient of the column plastic moment $\left(p_{c} \leq x_{A B}^{(A)} \leq 1\right)$ in collapse mechanism. In this column, by substituting $V_{A B}^{(A)}=s_{v, c} . V_{p, c}$ into the above equations, the minimum moment link length $\left(h_{m}\right)$ can be obtained as follows:

$$
\begin{aligned}
h_{m}= & \left(x_{B L}^{(A)}+x_{B R}^{(A)}+z \cdot\left(p_{c}+\left(1-p_{c}\right) \sqrt{1-s_{v, c}^{2}}\right)\right) \\
& \cdot \frac{\sqrt{3} h_{w, c}}{4 z \cdot\left(1-p_{c}\right) \cdot s_{v, c}} .
\end{aligned}
$$


By using $s_{v, c}=1$ in equation (30), maximum shear link length $\left(h_{v}\right)$ can be obtained as

$$
h_{v}=\left(x_{B L}^{(A)}+x_{B R}^{(A)}+z \cdot p_{c}\right) \frac{\sqrt{3} h_{w, c}}{4 z \cdot\left(1-p_{c}\right)} .
$$

For $h=h_{v}$, based on equation (29), $x_{A B}^{(A)}=p_{c}$, and consequently, $M_{A B}^{(A)}=M_{f, c}$; this state represents a short or shear link. Also, for $s_{v, c} \longrightarrow 0$, equation (30) gives $h_{m} \longrightarrow \infty$, and according to equation (29), we have $x_{A B}^{(A)}=1$, and consequently, $M_{A B}^{(A)}=M_{p, c}$, which indicates long link. This solution is valid within the framework of the static approach so that it provides actual internal reactions under yielding conditions in moment-shear interaction, and also, it can evaluate the corresponding plastic shear deformation $v_{P}^{(A)}$ and plastic rotation $\phi_{P}^{(A)}$. According to the normal plastic flow rule (equation (10)), the plastic shear deformation, $v_{P, A B}^{(A)}$ and the plastic rotation $\phi_{P, A B}^{(A)}$ for the central column can be obtained as follows:

$$
\begin{aligned}
v_{p, A B}^{(A)} & =2 \lambda \frac{V_{A B}^{(A)}}{V_{p, c}^{2}}, \\
\phi_{p, A B}^{(A)} & =2 \lambda \frac{M_{A B}^{(A)}-M_{f, c}}{M_{w, c}^{2}} .
\end{aligned}
$$

where, the parameter $\lambda$, which is controlling the large plastic flow, can be eliminated by dividing $\phi_{P}^{(A)}$ and $v_{P}^{(A)}$. By combining resultant equation with the kinematic compatibility conditions (equation (6)), the plastic shear deformation of the central column $v_{P, A B}^{(A)}$ and its plastic rotation $\phi_{P, A B}^{(A)}$ can be obtained as follows:

$$
v_{p, A B}^{(A)}=\theta h \frac{1}{1+\left(\left(\left(M_{A B}^{(A)}-M_{f, b}\right) / M_{w, b}^{2}\right)\left(V_{p, b}^{2} / V_{A B}^{(A)}\right)\right) h},
$$

$$
\phi_{p, A B}^{(A)}=\theta \frac{1}{1+\left(M_{w, b}^{2} /\left(M_{A B}^{(A)}-M_{f, b}\right)\right)\left(V_{A B}^{(A)} / V_{p, b}^{2} h\right)} .
$$

Finally, regarding this issue, the internal work $W_{i}^{(A)}$ can be obtained by substituting equations (11)-(13) in equation (3) as follows:

$$
W_{i}^{(A)}=M_{B L}^{(A)} \theta+M_{B R}^{(A)} \theta+M_{A B}^{(A)} \theta .
$$

Equations (35) shows that, in this type of mechanism, internal work in the beams and the central column of the TRF-H system can simply be expressed as $W_{\text {link }}=M^{(A)} \theta=M_{p \cdot e q} \theta$, where $M_{p \text { eq }}$ is yielding moment considering shear-moment interaction, that is, between $M_{f}$ and $M_{p}$. This result means that $M^{(A)}$ can be interpreted as an equivalent plastic moment, allowing to write the internal work simply on the basis of the equivalent plastic moment and the equivalent plastic rotation, even in the case of moment-shear interaction [14].

3.2. Mechanism Type " $B$ ". The relations required to solve the problem of moment-shear interaction in the intermediate links for the Mechanism " $B$ " are as follows.
(1) Condition for minimizing internal work of the " $B$ " type collapse mechanism under the influence of the rotation $\theta$ can be expressed as

$$
\operatorname{Min} \cdot W_{i}^{(B)}=2 M_{A B}^{(B)} \phi_{p, A B}^{(B)}+2 V_{A B}^{(B)} v_{p, A B}^{(B)} .
$$

(2) Kinematic compatibility condition is

$$
2 v_{p, A B}^{(B)}+\phi_{p, A B}^{(B)} h=\theta h .
$$

(3) Yielding condition of the link leads to the following function:

$$
F\left(M_{A B}^{(B)}, V_{A B}^{(B)}\right)=\left(\frac{\left|M_{A B}^{(B)}\right|-M_{f, c}}{M_{p, c}-M_{f, c}}\right)^{2}+\left(\frac{V_{A B}^{(B)}}{V_{p, c}}\right)^{2}-1
$$

(4) Normal plastic flow rule are as follows:

$$
\begin{aligned}
& v_{p}^{(B)}=\lambda \frac{\partial F}{\partial V^{(B)}}, \\
& \phi_{p}^{(B)}=\lambda \frac{\partial F}{\partial M^{(B)}},
\end{aligned}
$$

where $M_{A B}^{(B)}$ and $V_{A B}^{(B)}$ are, respectively, the bending moment and the shear created on the ends of the central column $(A B)$ of the TRF-H system in the mechanism " $B$." $\phi_{P}^{(B)}$ and $\nu_{P}^{(B)}$, respectively, are plastic rotation and plastic shear deformation created at the two ends of central column. For each member of the TRF-H system, the values of the shear and moment can be determined using the above relations. By substituting equation (37) into equation (36), the following equation is obtained:

$$
W_{i}^{(B)}=2 M_{A B}^{(B)} \phi_{p, A B}^{(B)}+V_{A B}^{(B)}\left(h \theta-h \Phi_{p, A B}^{(B)}\right) .
$$

For the certain value of $\theta$, the internal work relation can be expressed as a function of the plastic rotation of end of the columns $\phi_{P}^{(B)}$. So, minimizing the internal work could be expressed by

$$
\frac{\mathrm{d} W_{i}^{(B)}}{\mathrm{d} \phi_{p, A B}^{(B)}}=0 \longrightarrow V_{A B}^{(B)}=\frac{2 M_{A B}^{(B)}}{h} .
$$

By combining equation (38) with equation (41), the internal reactions in the central column of the TRF-H system can be determined as follows:

$$
\begin{aligned}
M_{A B}^{(B)} & =\frac{M_{f c}+M_{w, c} \sqrt{1-4\left(\left(M_{f, c}^{2}-M_{w, c}^{2}\right) / V_{p, c}^{2} h^{2}\right)}}{1+4\left(M_{w, c}^{2} / V_{p, c}^{2} h^{2}\right)}, \\
V_{A B}^{(B)} & =2 \frac{M_{f, c}+M_{w, c} \sqrt{1-4\left(\left(M_{f, c}^{2}-M_{w, c}^{2}\right) / V_{p, c}^{2} h^{2}\right)}}{h\left(1+4\left(M_{w, c}^{2} / V_{p, c}^{2} h^{2}\right)\right)} .
\end{aligned}
$$


Same as mechanism type " $A$," by substituting $M_{f, c}, M_{w, c}$, and $V_{p, c}$ into equations (42) and (43), the moment and shear value would be defined as a coefficient of the beam plastic moment:

$$
\begin{aligned}
& M_{A B}^{(B)}=x_{A B}^{(B)} \cdot M_{p, c}, \\
& V_{A B}^{(B)}=\frac{2 x_{A B}^{(B)}}{h} \cdot M_{p, c},
\end{aligned}
$$

where

$$
x_{A B}^{(B)}=\frac{p_{c}+\left(1-p_{c}\right) \sqrt{1-(3 / 4)\left(h_{w, c} / h\right)^{2}\left(\left(2 p_{c}-1\right) /\left(1-p_{c}\right)^{2}\right)}}{1+(3 / 4)\left(h_{w, c} / h\right)^{2}}, \quad p_{c} \leq x_{A B}^{(B)} \leq 1 .
$$

Using the above relations, for the different shear values $V=s_{v, c} \cdot V_{p, c}$, the moment multiplier can be obtained as follows:

$$
x_{B L}^{(A)}=p_{b}+\left(1-p_{b}\right) \sqrt{1-s_{c}^{2}},
$$

with respect to the above relation for a state, where $s_{c}=1$; according to equation (47), we have $x_{A B}^{(B)}=p_{c}$, and consequently, using equation (44), $M_{A B}^{(B)}=M_{f, c}$; this state indicates a short link. Also, for state where $s_{c} \longrightarrow 0$, equation (47) would conclude that $x_{A B}^{(B)}=1$; according to equation (44), $M_{A B}^{(B)}=M_{p, c}$, which indicates the long link.

Same as mechanism " $A$," plastic deformations $\nu_{P}^{(B)}$ and $\phi_{P}^{(B)}$ can be calculated at the ends of the link, depending on the kinematic conditions. Using the normal plastic flow rule, the parameter $\lambda$, which is controlling large plastic yield, could be eliminated by dividing $\phi_{P}^{(B)}$ and $v_{P}^{(B)}$. Then, by combining resultant equation with the kinematic compatibility, the plastic shear deformation of the central column $v_{P, A B}^{(B)}$ and its plastic rotation $\phi_{P, A B}^{(B)}$ can be obtained as follows:

$$
\begin{aligned}
& \phi_{p, A B}^{(B)}=\theta \frac{1}{1+\left(2 M_{w, c}^{2} /\left(M_{A B}^{(B)}-M_{f, c}\right)\right)\left(V_{A B}^{(B)} / V_{p, c}^{2} h\right)}, \\
& v_{p, A B}^{(B)}=\theta h \frac{1}{2+\left(\left(M_{A B}^{(B)}-M_{f, c}\right) / M_{w, c}^{2}\right)\left(V_{p, c}^{2} h / V_{A B}^{(B)}\right)} .
\end{aligned}
$$

\section{Hierarchy Criteria for TRF-H System}

4.1. Hierarchy Criteria for Intermediate Link Beams. In different mechanisms, three main elements of the TRF-H system could be as short, long, or intermediate links. The purpose of this research is to develop the hierarchy criteria for controlling the frame yield pattern of the one-storey TRF-H system. In the mechanism " $A$," all three elements of the TRF-H system have been yielded. In the mechanism " $B$," only the yield of the central column of this system has been considered. According to the kinematic theory of plastic collapse, the condition to achieve desired collapse mechanism is that the kinetic external force for a mechanism " $A$ " becomes less than the one for mechanism " $B$."

The internal virtual work is expressed for the mechanism “ $A$ ” using equation (35). For the mechanism " $B$, , $W_{i}^{(A)}$ can be obtained by substituting equation (41) in equation (40) as follows:

$$
B \text { - type mechanism: } W_{i}^{(B)}=2 M_{A B}^{(B)} \theta .
$$

External work is only due to the horizontal inertia force of the earthquake and is obtained for both the mechanisms by the following equation:

$$
W_{e, F}=F_{\text {ext }} \theta h .
$$

The coefficient of horizontal kinematic force is obtained by equating external work and internal work. Therefore, the following relation is obtained:

$$
\begin{aligned}
& A \text { - type mechanism: } F_{\text {ext }}^{(A)}=\frac{M_{B L}^{(A)}+M_{B R}^{(A)}+M_{A B}^{(A)}}{F h}, \\
& B \text { - type mechanism: } F_{\mathrm{ext}}^{(B)}=\frac{2 M_{A B}^{(B)}}{F h} .
\end{aligned}
$$

Given equations (52) and (53), it is possible to write a design criterion for the formation of the mechanism " $A$ " before the mechanism " $B$," for which the following relation must be satisfied:

$$
F_{\mathrm{ext}}^{(A)} \leq F_{\mathrm{ext}}^{(B)} \longrightarrow M_{B L}^{(A)}+M_{B R}^{(A)}+M_{A B}^{(A)} \leq 2 M_{A B}^{(B)} .
$$

Substituting equations (13) and (45) in equation (54) gives

$$
V_{A B}^{(A)} \leq V_{A B}^{(B)}
$$

In addition, substituting equations (17), (26), (27), and (44) in equation (54), the following relation is obtained:

$$
x_{B L}^{(A)}+x_{B R}^{(A)}+z \cdot x_{A B}^{(A)} \leq 2 z \cdot x_{A B}^{(B)} .
$$

4.2. Hierarchy Criteria for LongLinkBeams. In long links, the plastic shear deformation $\left(v_{p}\right)$ is negligible and is considered zero. Therefore, the internal work of the kinematic mechanisms discussed is easily obtained. In its mechanism " $A$ " (Figure 2(a)), three members of the TRF-H system including a two beams $B L$ and $B R$ and a column $A B$ would yield. Also, in its mechanism " $B$," the central vertical member of the TRF-H $(A B)$ system yields from both ends. In accordance 
TABLE 1: Characteristics of used profile sections.

\begin{tabular}{|c|c|c|c|c|c|}
\hline \multirow{2}{*}{ Model } & \multirow{2}{*}{ Element section } & \multicolumn{2}{|c|}{ Flange plate } & \multicolumn{2}{|c|}{ Web plate } \\
\hline & & $b_{f}(\mathrm{~mm})$ & $t_{f}(\mathrm{~mm})$ & $h_{w}(\mathrm{~mm})$ & $t_{w}(\mathrm{~mm})$ \\
\hline \multirow{2}{*}{ (I) } & Beam $(B L$ and $B R)$ & 200 & 10 & 20 & 6 \\
\hline & Column $(A B)$ & 200 & 20 & 200 & 8 \\
\hline \multirow{2}{*}{ (II) } & Beam $(B L$ and $B R)$ & 200 & 15 & 200 & 6 \\
\hline & Column $(A B)$ & 200 & 15 & 200 & 8 \\
\hline
\end{tabular}

with equations (18), (24), (29), and (46), for elements in different mechanisms, the value is $p \leq x \leq 1$. In each member, if the value $x=1$, the member is long and would have a bending behavior. Accordingly, by substituting this value in equation (56) for all the elements, the criteria for the different mechanism links can be obtained as follows:

$$
z \geq 2 \longrightarrow M_{p, c} \geq 2 M_{p, b} .
$$

4.3. Hierarchy Criteria for Short Link Beams. In short links, the moment and shear interaction according to Figure 2 is negligible, so only the shear plastic deformation is $v_{p}$ and the value of $\phi_{p}$ is zero according to the normal plastic flow rule. In accordance with equations (18), (24), (29), and (46), for each member in different mechanisms, if the value is $x=p$, the element is a short link and has shear behavior. Therefore, by substituting this value in equation (56), the hierarchy criteria for the short links can be obtained as follows:

$$
z \cdot p_{c} \geq 2 p_{b} \longrightarrow M_{f, c} \geq 2 M_{f, b} .
$$

\section{Numerical Examples and Verification}

In this section, in order to verify the hierarchy criteria obtained, these criteria are considered for the one-storey TRF-H frame, with particular attention to intermediate links in which the moment-shear interaction is important. The inelastic behavior of the designed structure has been examined with the pushover analysis to achieve the design goals, namely, the desired mechanism.

The profile of the T-part elements is shown in Table 1. The steel used in these members is S235 $\left(f_{y k}=235 \mathrm{MPa}\right)$ type. In order to validate the hierarchy criteria provided with intermediate links, two models are analyzed named model (I) and model (II).

In according to Eurocode 8 [1], links are classified as short links that have the following length of link:

$$
e \leq 1.6 \frac{M_{p, \text { link }}}{V_{p, \text { link }}},
$$

where $M_{p \text {,link }}=M_{f}=b_{f} t_{f} f_{y}\left(d-t_{f}\right)$ and $V_{p \text {,link }}=V_{p}=$ $\left(d-2 t_{f}\right) t_{w} f_{y} / \sqrt{3}$ are plastic moment and plastic shear in the link, respectively. It should be noted that, in equation (59), due to strain hardening, the flexural overstrength factor is about $1.2\left(\Omega_{m}\right)$ and the shear overstrength is equal to 1.5 $\left(\Omega_{v}\right)$. Therefore, using the equilibrium equation in the link, the maximum shear length in the ultimate state is as follows [34]:

$$
e=2.0 \frac{M_{u}}{V_{u}}=2.0 \frac{1.20 M_{p, \text { link }}}{1.50 V_{p, \text { link }}}=1.6 \frac{M_{p, \text { link }}}{V_{p, \text { link }}}
$$

where $M_{u}$ and $V_{u}$ are the ultimate moment and ultimate shear, respectively. Equation (60) is established for the links having two end rigid connections. In the TRF system with hinge connections, this equation can be used for the central column, but for the beams, the multiplier 1.6 is replaced by 0.8 . Obviously, these results could be easily generalized to the ultimate state using shear and flexural overstrength, as shown in Figure 2. With such an expansion of the plastic domain to reach the ultimate domain, all the relationships obtained in Sections 3 and 4 remain unchanged for the ultimate values of $M$ and $V$. Limitation provided by equation (60) for the elements of the TRF-H system with mentioned sections in Table 1, in model (I) for beam would be equal to 0.48 meters in the ultimate state and 0.61 meters in the plastic state and for the central column of the TRF-H system would be equal to 1.51 meters in the ultimate state and 0.94 meters in the plastic state. In Model (II) for beam would be equal to 0.74 meters in the ultimate state and 0.93 meters in the plastic state and for the central column of the TRF-H system would be equal to 0.69 meters in the ultimate state and 1.11 meters in the plastic state.

In models, (I) and (II), the span length is equal to $L=5.0 \mathrm{~m}$, the height of the storey is equal to $h=3.0 \mathrm{~m}$, and the length of the left beam is considered in the TRF-H system as an average link of $1.0 \mathrm{~m}\left(e_{L}=1.0 \mathrm{~m}\right)$. The moment-shear interaction diagram for cross section of the TRF-H system members is shown in Figure 4. In this figure, $B L$ and $B R$ correspond to the yielding of the left and right beams of the TRF-H system and $A B$ corresponds to the yielding of the central column of the TRF-H system.

It should be noted that, in design terms, plastic moments are reduced due to the effects of axial forces. This axial force could be easily calculated from the shear reaction results transmitted between the beam and the column of the TRF-H system and balanced by the axial forces of these elements.

To evaluate the validity of the hierarchy criteria previously described, various types of structures examined according to Table 1 were analyzed using pushover analyzes with the SAP2000 computer program [35]. It should be noted that, in the SAP2000 software, the moment-shear interaction could not be defined, and the elements of the TRF-H system are modeled as a beam and column element with a plastic hinge at their ends, in which the plastic moment is equal to the plastic moment, with the use of relationships in Section 4, has been determined. The mechanisms developed for the models examined are shown in Figure 5, where structural deformation is shown that these 


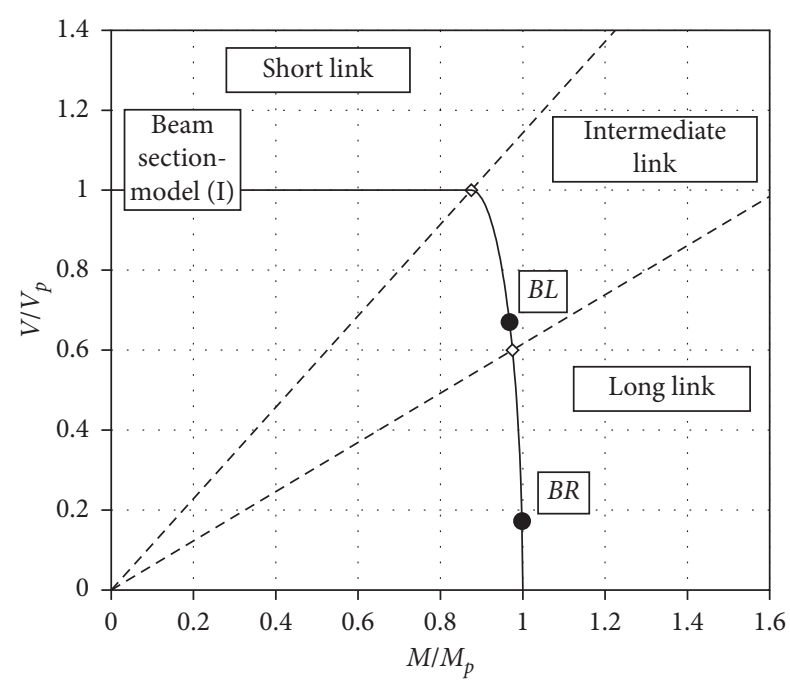

(a)

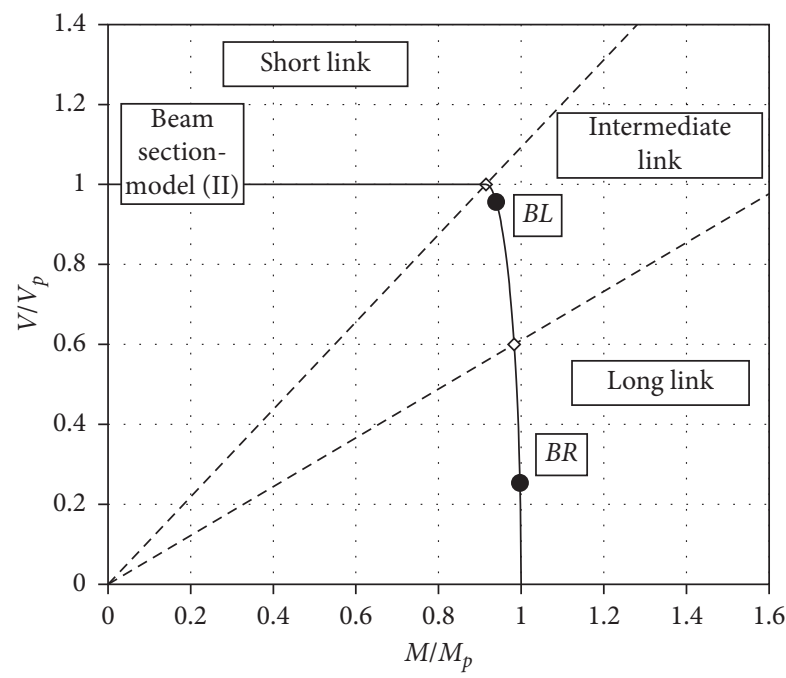

(c)

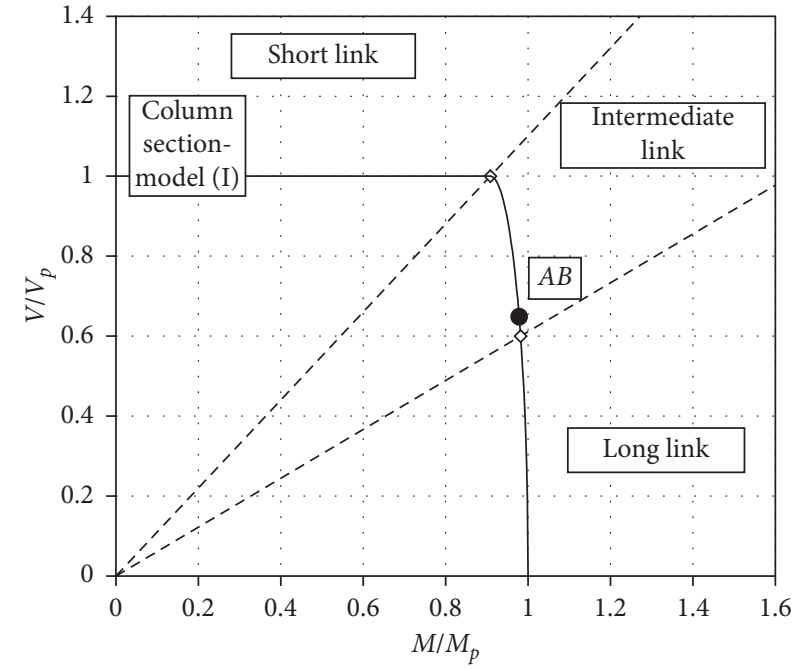

(b)

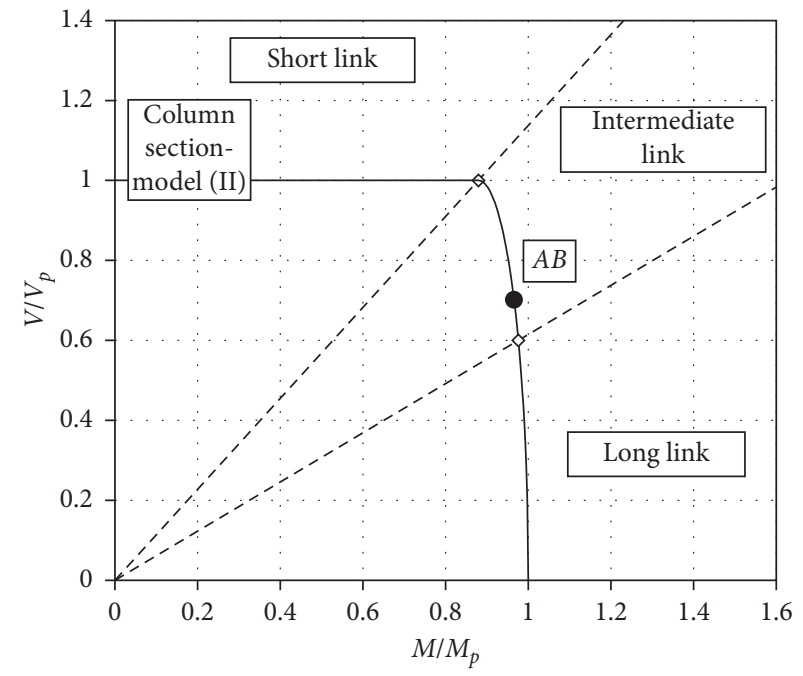

(d)

FIgURE 4: The moment-shear interaction diagram for T-part elements' sections. For the models studied, the results of the relationships given in Section 4 for determining the hierarchy criteria are shown in Table 2.

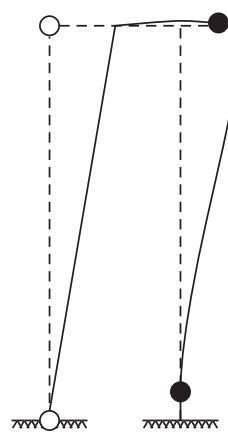

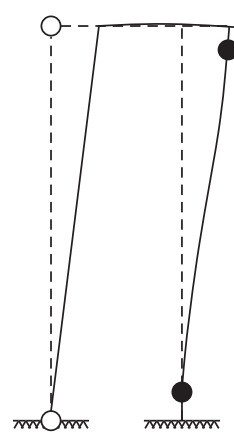

(a)

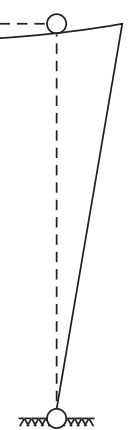

om (b)

FIgURE 5: Results of analysis of models (I) and (II). 
TABLE 2: The results of the moment-shear interaction and determining the type of mechanism.

\begin{tabular}{lcc}
\hline & Model (I) & Model (II) \\
\hline$x_{B L}^{(A)}$ & 0.9677 & 0.9398 \\
$x_{B R}^{(A)}$ & 0.9981 & 0.9972 \\
$x_{A B}^{(A)}$ & 0.9781 & 0.9653 \\
$x_{A B}^{(B)}$ & 0.9759 & 0.9826 \\
$x_{B L}^{(A)}+x_{B R}^{(A)}+z \cdot x_{A B}^{(A)}$ & 4.1193 & 3.0348 \\
$2 z \cdot x_{A B}^{(B)}$ & 4.2970 & 2.2351 \\
Mechanism & $(A)$ & $(B)$ \\
\hline
\end{tabular}

deformations are for the displacement value, which is arranged at the top of the storey to form a kinematic mechanism for all models examined, and there is a complete assurance.

Model (I) shown in Figure 5(a), according to the results of Table 2 and analysis, the collapse of the structure is in accordance with the Mechanism "A." In model (II) shown in Figure 5(b), according to the results of Table 2 and analysis, the collapse of the structure is in accordance with the Mechanism " $B$." The patterns obtained confirm the validity of the proposed design method.

\section{Conclusion}

In this paper, an analytical technique for considering the results of moment and shear interaction on the TRF-H system's intermediate links has been presented. The whole analysis was carried out in the framework of the rigid-plastic design using plastic domain diagram, normal flow rule, kinematic compatibility, and kinematic theory of plastic collapse to have a strong theoretical basis. In particular, the analytical relationships for the assessment of internal forces and the plastic deformation of intermediate links have been provided and the appropriate hierarchy criteria for the formation of the desired collapse mechanism have been obtained.

From the design point of view, the effect of link's strain hardening can be considered by applying the moment and shear overstrength factor in the plastic domain. The presented relationships are valid in the homothetic and nonhomothetic expansion of the plastic domain to determine the desired ultimate domain. The results obtained are required as a strong and applied theory in the capacity design principles for seismic design of TRF-H systems. In addition, the resultant hierarchy criteria lead to the development of a design method for failure mode control in multistorey TRF-H systems. This means that the final aim of the presented research is to ensure that a specific collapse mechanism has an expected yield hierarchy for the TRF-H system.

\section{Data Availability}

This article is based on mathematical relations. Modeling is compared with the results of mathematical relations, and its data are available on request from the authors.

\section{Conflicts of Interest}

The authors declare that they have no conflicts of interest.

\section{References}

[1] M. Bandehzadeh and P. Ashtari, "T-resisting frame concept: headway towards seismic performance improvement of steel frame," Journal of Constructional Steel Research, vol. 104, pp. 193-205, 2015.

[2] Bandehzadeh, "Evaluation of nonlinear behavior of steel buildings using deep beams as the lateral resistant system: Tbraced frame," M.Sc. thesis, Department of Civil Engineering, University of Zanjan, Zanjan, Iran, in Persian, 2009.

[3] M. Gorzin, R. Tajmir, and P. Ashtari, "Evaluation of endurance time method in seismic assessment of lateral T-resisting," Sharif Journal, Civil Engineering, vol. 2-31, no. 1/ 1, pp. 199-128, 2014.

[4] P. Ashtari, H. Barzegar, and F. Hamedi, "Experimental and numerical study on innovative seismic T-resisting frame (TRF)," Journal Structural Engineering and Mechanics, vol. 60, no. 2, pp. 251-269, 2016.

[5] P. Ashtari, H. Barzegar, and F. Hamedi, "Experimental and numerical evaluation of innovated T-resisting frames with haunched horizontal plate girders," Journal Advances in Structural Engineering, vol. 23, no. 8, 2020.

[6] CEN EN 1998-1-1, Eurocode 8-design of Structures for Earthquake Resistance. Part 1: General Rules, Seismic Actions and Rules for Buildings, Committee European de Normalisation CEN/TC 250, Brussels, Belgium, 2005.

[7] M. Bruneau, C. M. Uang, and A. Whittaker, Ductile Design of Steel Structures, McGraw Hill Professional, New York, NY, USA, 1997.

[8] C. W. Roeder and E. P. Popov, "Eccentrically braced steel frames for earthquakes," Journal of the Structural Division, vol. 104, no. 3, pp. 391-412, 1978.

[9] K. D. Hjelmstad and E. P. Popov, "Cyclic behavior and design of link beams," Journal of Structural Engineering, vol. 109, no. 10, pp. 2387-2403, 1983.

[10] K. Kasai and E. P. Popov, "General behavior of WF steel shear link beams," Journal of Structural Engineering, vol. 112, no. 2, pp. 362-382, 1986.

[11] T. Balendra, M.-T. Sam, C.-Y. Liaw, and S.-L. Lee, "Preliminary studies into the behaviour of knee braced frames subject to seismic loading," Engineering Structures, vol. 13, no. 1, pp. 67-74, 1991.

[12] D. C. Rai and B. J. Wallace, "Aluminium shear-links for enhanced seismic resistance," Earthquake Engineering \& Structural Dynamics, vol. 27, no. 4, pp. 315-342, 1998.

[13] P. Dusicka, AM. Itani, and I. G. Buckle, "Evaluation of conventional and specialty steels in shear link hysteretic energy dissipaters," in Proceedings of the 13th WCEE, Vancouver, Canada, August 2004.

[14] L. Mastrandrea, R. Montuori, and V. Piluso, "Shear-moment interaction in plastic design: eccentrically braced frames," in Proceedings of the 4th International Conference on Behavior of Steel Structures in Seismic Areas, Naples, Italy, June 2003.

[15] R. Montuori, E. Nastri, and V. Piluso, "Rigid-plastic analysis and moment-shear interaction for hierarchy criteria of inverted Y EB-frames," Journal of Constructional Steel Research, vol. 95, pp. 71-80, 2014.

[16] L. Mastrandrea, R. Montuori, and V. Piluso, "Failure mode control of seismic resistant EB frames," in Proceedings of the 
4th International Conference on Behavior of Steel Structures in Seismic Areas NAPLES, Naples, Italy, June 2003.

[17] MA. Conti, L. Mastrandrea, and V. Piluso, "Plastic design and seismic response of knee braced frames," Advance Steel Construction, vol. 5, no. 3, pp. 343-366, 2009.

[18] M. T. Giugliano, A. Longo, R. Montuori, and V. Piluso, "Failure mode and drift control of MRF-CBF dual systems," The Open Construction \& Building Technology Journal, vol. 4, no. 1, pp. 121-133, 2010.

[19] A. Longo, R. Montuori, and V. Piluso, "Theory of plastic mechanism control of dissipative truss moment frames," Engineering Structures, vol. 37, pp. 63-75, 2012.

[20] R. Montuori, E. Nastri, and V. Piluso, "Theory of plastic mechanism control for MRF-EBF dual systems: closed form solution," Engineering Structures, vol. 118, pp. 287-306, 2016.

[21] R. Montuori, E. Nastri, and V. Piluso, "Seismic response of EB-frames with inverted Y-scheme: TPMC versus Eurocode provisions," Earthquakes and Structures, vol. 8, no. 5, pp. 1191-1214, 2015.

[22] T. Okazaki, M. D. Engelhardt, M. Nakashima, and K. Suita, "Experimental study on link to column connections in steel eccentrically braced frames," in Proceedings of the 13th World Conference on Earthquake Engineering, Vancouver, Canada, August 2004.

[23] AISC, Seismic Provisions for Structural Steel Buildings, American Institute of Steel Construction Standard ANSI/ AISC 341-10, Chicago, IL, USA, 2010.

[24] T. Okazaki, M. D. Engelhardt, A. Drolias, E. Schell, J.-K. Hong, and C.-M. Uang, "Experimental investigation of link-to-column connections in eccentrically braced frames," Journal of Constructional Steel Research, vol. 65, no. 7, pp. 1401-1412, 2009.

[25] B. G. Neal, "Effect of shear force on the fully plastic moment of an I-beam," Journal of Mechanical Engineering Science, vol. 3, no. 3, pp. 258-266, 1961.

[26] K. D. Hjelmstad and E. P. Popov, "Seismic behavior of active beam link in eccentrically braced frames," EERC Report No. 83-15, Earthquake Engineering Research Center, University of California, Berkeley, CA, USA, 1983.

[27] J. O. Malley and E. P. Popov, "Design consideration for shear links in eccentrically braced frames," EERC Report No. 83-24, Earthquake Engineering Research Center, University of California, Berkeley, CA, USA, 1983.

[28] CEN EN 1993-1-1, Eurocode 3: Design of Steel Structures. Part 1: General Rules and Rules for Buildings, Committee European de Normalization CEN/TC 250, Brussels, Belgium, 2005.

[29] E. P. Popov and M. D. Engelhardt, "Seismic eccentrically braced frames," Journal of Constructional Steel Research, vol. 10, pp. 321-354, 1988.

[30] G. Della Corte, M. D’Aniello, and R. Landolfo, "Analytical and numerical study of plastic overstrength of shear links," Journal of Constructional Steel Research, vol. 82, pp. 19-32, 2013.

[31] E. Barecchia, G. Della Corte, and F. M. Mazzolani, "Plastic over-strength of short and intermediate links," in Proceedings of the STESSA 2006 Conference on Behavior of Steel Structures in Seismic Areas, pp. 177-183, Yokohama, Japan, August 2006.

[32] American Association of State and Highway Transportation Officials AASHTO, AASHTO LRFD Bridge Design Specifications, AASHTO, Washington, DC, USA, 2nd edition, 1998.

[33] American Institute of Steel Construction (AISC), Load and Resistance Factor Design Specification for Structural Steel Buildings, American Institute of Steel Construction, Chicago, IL, USA, 1999.
[34] M. D. Engelhardt and E. P. Popov, "Behavior of long links in eccentrically braced frames," EERC Report No. 89-01, Earthquake Engineering Research Center, University of California, Berkeley, CA, USA, 1989.

[35] CSI SAP, Integrated finite Element Analysis and Design of Structures. Analysis Reference, Computer and Structure Inc. University of California, Berkeley, CA, USA, 2000. 